\title{
CONVEXITY PROPERTIES OF NONLINEAR MAXIMAL MONOTONE OPERATORS
}

\author{
BY R. T. ROCKAFELLAR ${ }^{1}$
}

Communicated by J. J. Kohn, June 27, 1968

Let $X$ be a real Banach space with dual $X^{*}$. A monotone operator from $X$ to $X^{*}$ is by definition a (generally multivalued) mapping $T$ such that

$$
\left\langle x-y, x^{*}-y^{*}\right\rangle \geqq 0 \quad \text { whenever } \quad x^{*} \in T(x), \quad y^{*} \in T(y)
$$

(where $\langle\cdot, \cdot\rangle$ denotes the pairing between $X$ and $X^{*}$ ). Such an operator is said to be maximal if there is no monotone operator $T^{\prime}$ from $X$ to $X^{*}$, other than $T$ itself, such that $T^{\prime}(x) \supset T(x)$ for every $X$. The effective domain $D(T)$ and range $R(T)$ of a monotone operator $T$ are defined by

$$
\begin{aligned}
& D(T)=\{x \mid T(x) \neq \varnothing\} \subset X, \\
& R(T)=U\{T(x) \mid x \in X\} \subset X^{*} .
\end{aligned}
$$

Minty [9] has shown that, when $X$ is finite-dimensional and $T$ is a maximal monotone operator, the sets $D(T)$ and $R(T)$ are almost convex, in the sense that each contains the relative interior of its convex hull. The purpose of this note is to announce some generalizations of Minty's result to infinite-dimensional spaces.

A subset $C$ of $X$ will be called virtually convex if, given any relatively (strongly) compact subset $K$ of the convex hull of $C$ and any $\epsilon>0$, there exists a (strongly) continuous single-valued mapping $\phi$ from $K$ into $C$ such that $\|\phi(x)-x\| \leqq \epsilon$ for every $x \in K$. It can be shown that, in the finite-dimensional case, $C$ is virtually convex if and only if $C$ is almost convex, so that the following result contains Minty's result as a special case.

THEOREM 1. Let $X$ be reflexive, and let $T$ be a maximal monotone operator from $X$ to $X^{*}$. Then the strong closures of $D(T)$ and $R(T)$ are convex. If in addition $X$ is separable, or if $X$ is an $L^{p}$ space with $1<p<\infty, D(T)$ and $R(T)$ are virtually convex.

The proof of Theorem 1, which will appear in [12], is made possible by recent results of Asplund [1], [2] concerning the existence of

1 Supported in part by the Air Force Office of Scientific Research under grant AF-AFOSR-1202-67. 
single-valued duality mappings $J: X \rightarrow X^{*}$, and results of Browder [5] concerning the invertibility of mappings of the form $T+\lambda J, \lambda>0$, where $T$ is a maximal monotone operator.

Since the subdifferential $\partial f$ of a lower semicontinuous proper convex function $f$ on $X$ is a maximal monotone operator (Rockafellar [14]), Theorem 1 yields a new result about the existence of subgradients. (It has been observed elsewhere [3] that the strong closures of $D(\partial f)$ and $R(\partial f)$ are convex even when $X$ is not reflexive.)

CoRollary. Let $X$ be reflexive and separable (or an $L^{p}$ space with $1<p<\infty)$, and let $f$ be a lower semicontinuous proper convex function on $X$. Then $D(\partial f)$ and $R(\partial f)$ are virtually convex.

Theorem 1 is applicable in particular to any single-valued monotone operator $T$ with $D(T)=X$ such that $T$ is hemicontinuous, i.e. continuous from line segments in $X$ to the weak* topology of $X^{*}$, since such a $T$ is known to be maximal (Browder [4]).

The following convexity result covers certain cases where $X$ is not reflexive. Here $T$ is said to be locally bounded at a point $x$ if there exists a neighborhood $U$ of $x$ such that the set

$$
T(U)=U\{T(u) \mid u \in U\}
$$

is bounded in $X^{*}$.

THEOREM 2. Let $T$ be a maximal monotone operator from $X$ to $X^{*}$. Suppose either that the convex hull of $D(T)$ has a nonempty interior, or that $X$ is reflexive and there exists a point of $D(T)$ at which $T$ is locally bounded. Then the interior of $D(T)$ is a convex set whose (strong) closure is the closure of $D(T)$. Moreover, $T$ is locally bounded at every interior point of $D(T)$, whereas $T$ is not locally bounded at any boundary point of $D(T)$.

The local boundedness assertion of Theorem 2 strengthens a result of Kato [8], according to which a monotone operator $T$ is locally bounded at any interior point of $D(T)$ where it is hemibounded.

Theorem 2 will be deduced in [13] from a more general theorem for locally convex spaces. The theorem of Debrunner-Flor [6] plays an important role in the proof.

The consequences of Theorem 2 include:

CoRollary 1. Let $X$ be reflexive, and let $T$ be a maximal monotone operator from $X$ to $X^{*}$ such that the convex hull of $R(T)$ has a nonempty interior. Then the interior of $R(T)$ is a convex set whose closure is the (strong) closure of $R(T)$. 
Corollary 2. Let $T$ be a maximal monotone operator from $X$ to $X^{*}$, and let $D_{0}$ be the subset of $D(T)$ where $T$ is single-valued. Then $T$ is demicontinuous on $D_{0}$, i.e. continuous as a single-valued mapping from $D_{0}$ in the strong topology to $X^{*}$ in the weak* topology.

Corollary 3. Let $X$ be reflexive, and let $T$ be a maximal monotone operator from $X$ to $X^{*}$. Suppose there exists a subset $B$ of $X$ such that 0 belongs to the interior of the convex hull of

$$
T(B)=U\{T(x) \mid x \in B\} .
$$

Then there exists an $x$ such that $0 \in T(x)$.

Corollary 4. Let $X$ be reflexive, and let $T$ be a maximal monotone operator from $X$ to $X^{*}$. In order that $R(T)$ be all of $X^{*}$, it is necessary and sufficient that, whenever $x_{i}^{*} \in T\left(x_{i}\right)$ for $i=1,2, \cdots$, and $\left\|x_{i}\right\| \rightarrow \infty$, then the sequence $x_{1}^{*}, x_{2}^{*}, \cdots$, has no strongly convergent subsequence.

Corollary 2 may be compared with the result of Kato [8] that a single-valued monotone operator $T$ is demicontinuous on any open subset of $D(T)$ where it is hemicontinuous. Corollary 3 is a generalization of the main existence theorem of Minty [10], which requires in effect that 0 be an interior point of the convex hull $T_{0}(B)$, where $T_{0}$ is some mapping such that $T_{0}(x) \subset T(x)$ for every $x$ and

$$
\sup _{x \in \boldsymbol{B}} \sup _{x^{*} \in T_{0(x)}}\left\langle x, x^{*}\right\rangle<\infty .
$$

The necessary and sufficient condition in Corollary 4 is satisfied, in particular, when the following condition is satisfied: whenever

and

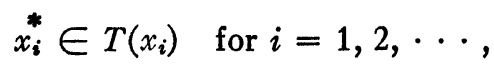

$$
\lim _{i \rightarrow \infty}\left\|x_{i}\right\|=\infty \text {, then } \lim _{i \rightarrow \infty}\left\|x_{i}^{*}\right\|=\infty .
$$

(The two conditions are equivalent, of course, when $X$ is finitedimensional.) The sufficiency of the latter condition for $R(T)$ to be all of $X^{*}$ has previously been established by Browder [4, Theorem 4].

\section{REFERENCES}

1. E. Asplund, Averaged norms, Israel J. Math. 5 (1967), 227-233.

2. - Positivity of duality mappings, Bull. Amer. Math. Soc. 73 (1967), 200-203.

3. A. Brondsted and R. T. Rockafellar, On the subdifferentiability of convex functions, Proc. Amer. Math. Soc. 16 (1965), 405-411.

4. F. E. Browder, Multivalued monotone nonlinear mappings and duality mappings in Banach spaces, Trans. Amer. Math. Soc. 118 (1965), 338-351. 
5. - Nonlinear maximal monotone operators in Banach spaces, Math. Ann. 175 (1968), 89-113.

6. H. Debrunner and P. Flor, Ein Erweiterungssatz für monotone Mengen, Arch. Math. 17 (1964), 445-447.

7. T. Kato, Demicontinuity, hemicontinuity and monotonicity, Bull. Amer. Math. Soc. 70 (1964), 548-550.

8. - Demicontinuity, hemicontinuity and monotonicity. II, Bull. Amer. Math. Soc. 73 (1967), 886-889.

9. G. J. Minty, On the maximal domain of a 'monotone' function, Michigan Math. J. 8 (1961), 135-137.

10. - On the solvability of nonlinear functional equations of 'monotonic' type, Pacific J. Math. 14 (1964), 249-255. (A correction to the maximality proof in this paper is given in [14].)

11. R. T. Rockafellar, Characterization of the subdifferentials of convex functions, Pacific J. Math. 17 (1966), 497-510.

12. - On the virtual convexity of the domain and range of a nonlinear maximal monotone operator, Math. Ann. (to appear).

13. - Local boundedness of nonlinear monotone operators, Michigan Math. J. (to appear).

14. - On the maximal monotonicity of subdifferential mappings, Michigan Math. J. (to appear).

University of Washington, Seattle, Washington 98105 